\section{Valsalva Maneuver}

Editor - I enjoyed the article "Antonio Maria Valsalva $(1666-1723) " 1$ and would like to add that as well as diagnosing cardiac conditions, the Valsalva maneuver is also part of the autonomic testing battery. There are two specific autonomic components:

1. Valsalva ratio: this is the ratio of heart rate phase IV/heart rate phase II, and is essentially a measure of vagal function. The normal response is an increase in heart rate during phase II in response to the fall in blood pressure, and the baroreflex response to the blood pressure overshoot in phase IV is transient bradycardia. In dysautonomic patients, there is typically a loss of both the blood pressure overshoot and the reflex bradycardia. Age-adjusted normal Valsalva ratios are used as controls for the Valsalva ratio.

2. Beat-to-beat blood pressure response to the Valsalva maneuver: phase II may be divided into early and late phases. In early phase II, reduced preload and stroke volume lead to a fall in cardiac output despite the tachycardia caused by decreased vagal activity. Total peripheral resistance increases due to increased sympathetic discharge (nucleus tractus solitarius) and an increase in plasma epinephrine concentration. The fall in blood pressure is thus arrested, and is termed "late phase II"; in normal patients there is actually a rise in blood pressure just before release. Interestingly, this phase can be blocked with alpha-antagonists (e.g., phentolamine) while phase IV can be blocked with beta-blockers.

Patients with peripheral adrenergic failure due to involvement of autonomic fibers will have absent late phase II and in fact can have such dramatic drops in blood pressure during forced expiration that syncope may occur.

1. Yale SH. Antonio Maria Valsalva (1666 - 1723). Clinical Medicine \& Research 2005;3:35-38.

Bradley C. Hiner, MD Department of Neurology, Marshfield Clinic Marshfield, Wisconsin

\section{Author's Reply}

Editor - Dr. Hiner's comments regarding the use of the Valsalva maneuver in the comprehensive assessment of the cardiovagal (Valsalva ratio) and adrenergic (blood pressure) functions of the autonomic nervous system are appreciated. The discussion in the article on Antonio Maria Valsalva ${ }^{1}$ was focused on the use of the Valsalva maneuver as part of the bedside technique for evaluating heart murmurs and heart failure; however, Dr. Hiner's description of the Valsalva ratio and beat-to-beat blood pressure response in autonomic testing illustrates the laboratory applications of this technique.

In addition, the Valsalva maneuver has been used in multiple disciplines. For example, the use of the Valsalva maneuver during Doppler ultrasound examinations helps to improve the identification of patients with abnormalities such as lower extremity venous insufficiency, diastolic dysfunction, and patent foramen ovale. ${ }^{2}$ In urological examinations, the Valsalva maneuver is used to determine urethral competence (sphincter insufficiency) in patients with stress urinary incontinence 3 and helps to identify varicoceles during male infertility testing. ${ }^{4}$

The Valsalva maneuver can also be used to assist in identifying lesions in the head and neck when they are not well delineated due to tumors abutting against mucosal surfaces. ${ }^{5}$ Other serious and life threatening complications have been identified by using the Valsalva maneuver. These include calvarial and upper cervical hyperpneumatization, pneumothorax, pneumomediastinum, and pneumoperitoneum, retinal, macular, and vitreous hemorrhages, and retinal vein occlusion. $6,7,8$

1. Yale SH. Antonio Maria Valsalva (1666 - 1723). Clinical Medicine \& Research 2005;3:35-38.

2. Khouri SJ, Maly GT, Suh DD, Walsh TE. A practical approach to the echocardiographic evaluation of diastolic function. J Am Soc Echocardiogr 2004;17:290-297.

3. Daneshgari F. Valsalva leak point pressure: steps toward standardization. Curr Urol Rep 2001;2:388-391.

4. Belker AM. The varicocele and male infertility. Urol Clin North Am 1981;8:41-51.

5. Henrot P, Blum A, Toussaint B, Troufleau P, Stines J, Roland J. Dynamic maneuvers in local staging of head and neck malignancies with current imaging techniques: principles and clinical applications. Radiographics 2003;23:1201-1213.

6. Littrell LA, Leutmer PH, Lane JI, Driscoll CL. Progressive calvarial and upper cervical pneumatization associated with habitual valsalva maneuver in a 70-year-old man. AJNR Am J Neuroradiol 2004;25:491-493.

7. Chapman-Davies A, Lazarevic A. Valsalva maculopathy. Clin Exp Optom 2002;85:42-45.

8. Kadrmas EF, Pach JM. Vitreous hemorrhage and retinal vein rupture. Am J Ophthalmol 1995;120:114-115. 
Steven Yale, MD, FACP Department of Internal Medicine, Marshfield Clinic Director of Clinical Research, Marshfield Clinic Research Foundation Marshfield, Wisconsin

\section{Sensitivity and Specificity}

Editor - In the Authors' Reply to the letter "Sensitivity and Specificity of Tests of Liver Injury" in the November 2004 issue of Clinical Medicine \& Research, the statement "It is true that no single test of liver injury is either highly sensitive or specific" would be more accurate if it read "It is true that no single test of liver injury is both highly sensitive and specific". It is easy to come up with a test (for anything) that is either perfectly sensitive or perfectly specific; the test is of little use without some degree of both.

1. Musana KA, Yale SH, Abdulkarim AS. Authors' reply: Sensitivity and specificity of tests of liver injury. Clinical Medicine \& Research 2004;2:205.

Richard Berg, MS Biostatistics and Bioinformatics Core, Marshfield Clinic Research Foundation Marshfield, Wisconsin
The name and credentials of Dr. James Freeman were incorrectly listed in the 2004 Reviewer Index that appeared in the November 2004 issue of Clinical Medicine \& Research (2004;2:279-281). The correct listing should read "James R. Freeman, DO. Marshfield Clinic - Eau Claire Center. Eau Claire, WI, USA". We sincerely apologize for these errors.

Editorial Staff Clinical Medicine \& Research 